\title{
A Hybrid Multi-Domain Index Modulation for Covert Communication
}

\author{
Xi Yao, Ping Yang, Senior Member, IEEE, Jialiang Fu, Yue Xiao, Member, IEEE, and Zilong Liu, Member,
} IEEE

\begin{abstract}
In this letter, a hybrid multi-domain index modulation scheme based on spreading codes domain and beam domain is proposed. In our design, the information bit stream is divided into two parts: one for covert transmission using the index of spreading code and the other using the directional modulation to improve bit error ratio (BER) performance in the desired direction and to prevent eavesdropping. Then, based on joint boundaries and statistical theory, the average error probabilities of legitimate users and eavesdroppers are derived. Moreover, we analyze the average BER and validate through simulation results that the proposed hybrid multi-domain scheme is capable of achieving better BER performance compared to conventional coded wireless communication systems.
\end{abstract}

Index Terms-Covert communication, directional modulation, index modulation

\section{INTRODUCTION}

I NDEX modulation (IM) [1]-[4] is an attractive approach to achieve enhanced security, in which the indices of the transmitting antenna [1], subcarriers [2], time intervals, spreading codes and pre-coded matrices are employed for information transmission. Among the current research of IM used for covert communication, secure spatial modulation (SM) schemes have attracted many attentions [5]-[7], which exploits the antenna jamming to conceal information. A new IM technique, namely code-index modulation (CIM) based spread spectrum, was proposed in [8], which uses the index of the spreading code to carry additional information besides the the transmitted symbols. Then, [9]-[11] further proposed generalized combinations of code index modulation and spatial modulation (or its variants, e.g., quadrature spatial modulation). As a further extension, [12] proposed a novel CIM design based on two-way network coding techniques.

Recently, directional modulation (DM) [13] with the aid of phased array has also attracted increasing research attention. The key idea of DM is to employ a phase shifter to generate the correct phase and

X. Yao, J. Fu, P. Yang, and Y. Xiao are with the National Key Laboratory of Science and Technology on Communications, University of Electronic Science and Technology of China, 611731, Sichuan, China. X. Yao and P. Yang are also with the State Key Laboratory of Integrated Services Networks, Xidian University. (e-mail: 201821220261@std.uestc.edu.cn, 1611251640@qq.com, yang.ping@ uestc.edu.cn, xiaoyue@uestc.edu.cn).

Z. Liu is with the School of Computer Science and Electronics Engineering, University of Essex, UK((zilong.liu@essex.ac.uk).

Corresponding author: P. Yang (e-mail: yang.ping@uestc.edu.cn).

This work is supported by the National Science Foundation of China under Grant number 61876033, the National Science Foundation of China under Grant number U19B2014 and the Open Project of State Key Laboratory of Integrated Services Networks of Xidian University under Grant number ISN21-14. amplitude in given direction whilst distorting the signal in other directions, so as to achieve safe transmissions. Traditional phased array beamforming [14] transmits the identical signal in all directions, depending only on the azimuth of the destination, instead of the range. Frequency division arrays (FDA) [15]-[18] were used as an effective technique capable of generating distance-dependent patterns. For example, the work in [19] and [20] used the uniform element spacing and linearly increasing frequency increments to achieve secure communications at the physical layer. Moreover, both uniform element spacing, as well as symmetric and non-linearly increasing frequency increments were utilised in [21] to separate the angle and distance-dependent transmission beam maps of FDA and DM aided secure signal transmissions. In addition, [22] proposed a DM scheme based on a random frequency diversity array by incorporating artificial noise.

Although IM naturally has a covert transmission feature relying on the index information of different domains, it is subject to eavesdropping in lost of sight (LoS) channels. By observing the fact that both DM and IM enable secure transmissions, spatial and directional modulation (SDM) with scrambling was studied in [23], in which each modulation symbol is phase scrambled. In particular, by combining SM [24] and DM, the resulting system provided enhanced safety in addition to a higher spectral efficiency. In this letter, we propose a new system, called code-domain index modulation with DM (CDIDM), which combines the advantages of CIM and DM to achieve high spectral efficiency and information security.

Specifically, compared to the spatial-domain based designs [23] [24], we extend the design framework to code-domain and propose a hybrid multi-domain index modulation by combining coding index to carry information with DM. We present the maximum likelihood (ML) scheme for Bob and Eve receivers and derive their average BER bounds. Our simulation results show that the system is able to achieve an angle concealment of about $10^{\circ}$ which considerably outperforms the conventional system.

Notation: $C(n, k),\|\cdot\|$ and $\mathbb{E}[\cdot]$ denote the binomial coefficient, two norm of a matrix or vector, and expectation operation, respectively. $\operatorname{Tr}[\cdot]$ represents the trace of a matrix. $(\cdot)^{T}$ and $(\cdot)^{H}$ indicate the transpose and Hermitian transpose of a matrix or vector, respectively. $\operatorname{det}|\cdot|$ is the determinant of a matrix. 


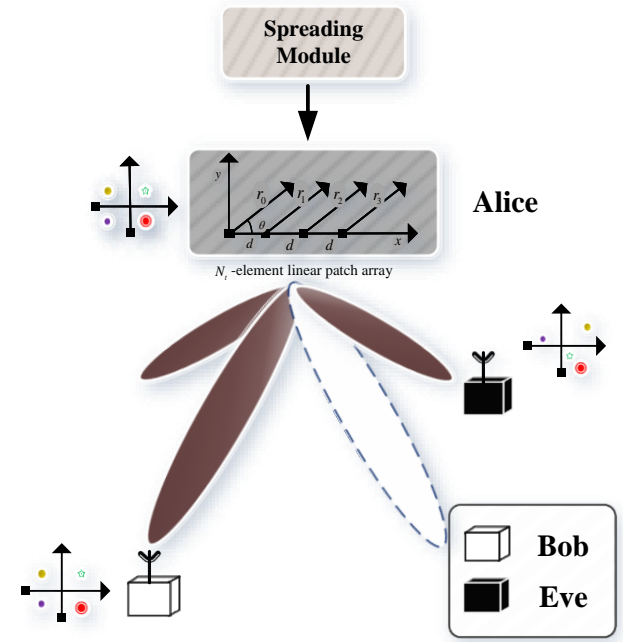

Fig. 1. System model of the CDIDM

\section{Code-Domain Index And DiRectional Modulation}

We first introduce the system model of the CDIDM scheme and then detail the transmitter architecture. In our design, the transmit information are concealed by jointly employing the index of the spreading codes and the beam designed. Since more hiding parts are utilized and multi-domain are exploited, the proposed CDIDM scheme is capable of achieving excellent security performance.

\section{A. System Model}

As shown in Fig. 1, our system consists of one spreading module used for spread code selection and spreading, one $N_{t}$-element linear antenna array at the transmitter (Alice), a single antenna receiver at the legitimate user (Bob) and a single antenna at the eavesdropper (Eve). Particularly, based on the concepts of DM, Alice projects the modulated signal onto receivers, as shown in Fig. 1, where the constellation signals can be correctly received at Bob, while the signals are scrambled at Eve.

We use the LoS channel model which is widely used in DM studies. A uniform line array (ULA) is adopted by Alice, and a phase reference is set at the geometric center of the array. As a result, the channel coefficients of the receiver located along the direction $\theta$ can be expressed as $\mathbf{h}^{H}(\theta)=\left[e^{-j N_{c} L(\lambda, d, \theta)}, e^{-j\left(N_{c}-1\right) L(\lambda, d, \theta)}, \ldots, e^{j N_{c} L(\lambda, d, \theta)}\right]$,

where $N_{c}=\left(\frac{N_{t}-1}{2}\right)$ is the center of the antenna array, $L(\lambda, d, \theta)=\frac{2 \pi}{\lambda} d \cos \theta$ is a function of the wavelength $\lambda$, antenna spacing $d$ at Alice, and direction $\theta$ at Bob. Specially, in order to avoid the creation of grating lobs, we assume $d \leq \lambda / 2$. Thus, the downstream channel matrix between Alice and Bob is expressed as

$$
\mathbf{H}\left(\Theta_{B}\right)=\left[\mathbf{h}\left(\theta_{1}\right), \mathbf{h}\left(\theta_{2}\right), \ldots, \mathbf{h}\left(\theta_{N_{r}}\right)\right]^{H},
$$

where $\Theta_{B}=\left\{\theta_{1}, \ldots, \theta_{N_{r}}\right\}$ represents the set of the directions of Bob, while we have $\mathbf{H}\left(\Theta_{B}\right) \in \mathbb{C}^{N_{r} \times N_{t}}$ and $\operatorname{rank}\left(\mathbf{H}\left(\Theta_{B}\right)\right)=N_{t}$.

\section{B. Specific Transmitter Architecture}

The specific CDIDM transmitter architecture is depicted in Fig. 2. The receiver uses ML detection to detect code index and DM signal respectively. Prior to transmission via the $N_{t}$-element linear antenna array $\left(N_{t}=4\right.$ for example), the transmission bits of the CDIDM scheme in a frame is divided into two parts: one part is used for mapping to $M$-PSK/QAM symbols, while the other is mapped to the indices of the spreading codes.

Assume that there are $N$ spread codes to choose from in the spread code set. For each transmission, one of the spread codes is selected based on $\left\lfloor\log _{2}(N)\right\rfloor$ information bits. In addition, it takes $\log _{2}(M)$ bits to transfer a $M$-PSK/QAM symbol from a modulation alphabet $\mathcal{B}$ $(|\mathcal{B}|=M)$. Thus, CDIDM achieves a rate of $\left\lfloor\log _{2}(N)\right\rfloor+$ $\log _{2}(M)$ bits per channel. After code index modulation, the CDIDM symbol vector can be expressed as

$$
s_{i}^{m}=c_{i} b_{m},
$$

where $\boldsymbol{c}_{i}=\left[c_{i, 1}, c_{i, 2}, \ldots c_{i, k}\right]^{T}$, which represents the $i$ th spreading code activated from $N$ spreading codes for Bob, $k$ is the length of the spreading code, and $b_{m} \in \mathcal{B}$ $\left(\mathcal{B}=\left\{b_{1}, b_{2}, \ldots, b_{M}\right\}\right)$ is the $M$-PSK/QAM symbol, which satisfies either the loose power constraint of $\mathbb{E}\left({ }_{\mid} b_{m}{ }^{2} \mid\right)=1$ or the strict power constraint of $\left|b_{m}\right|^{2}=1$. For further hiding the information transmitted by Alice, the DM technique based on precoding is employed and the corresponding generated signal vector is

$$
\mathbf{x}=\mathbf{P} s_{i}^{m}
$$

where $\mathbf{P} \in \mathbb{C}^{N_{t} \times k}$ is the precoding matrix. In the proposed DM scheme, $\mathbf{P}=\left[\mathbf{p}_{1}, \mathbf{p}_{2}, \ldots, \mathbf{p}_{\mathrm{k}}\right]$ is designed to ensure that only Bob can correctly receive the modulated signal. Therefore, the precoding matrix $\mathbf{P}$ can be expressed as

$$
\mathbf{P}=\mathbf{H}^{H}\left(\Theta_{B}\right)\left(\mathbf{H}\left(\Theta_{B}\right) \mathbf{H}^{H}\left(\Theta_{B}\right)\right)^{-1} .
$$

\section{Signal Detection}

1) Bob's Dectection: The transmitted CDIDM signal in (4) passes through the LoS channel, and thus the received signal at any direction $\Theta_{B}$ can be expressed as

$$
\begin{aligned}
\mathbf{y}_{B} & =\mathbf{H}^{H}\left(\Theta_{B}\right) \mathbf{x}+\mathbf{n} \\
& =\boldsymbol{c}_{i} b_{m}+\mathbf{n},
\end{aligned}
$$

where $\mathbf{n} \sim \mathcal{C N}\left(0, \sigma_{n}^{2}\right)$ is the additive white Gaussian noise (AWGN) vector.

Based on (6), the ML detector is employed to jointly detect $b_{m}$ and $i$, which is given by

$$
\left\langle\hat{i}, \hat{b}_{m}\right\rangle=\arg \min _{\substack{b_{m} \in \mathcal{B} \\ i \in\{1,2, \ldots, N\}}}\left\|\mathbf{y}_{B}-\boldsymbol{c}_{i} b_{m}\right\|^{2},
$$

where $\hat{i}$ represents the estimated index of the selected spreading code and $\hat{b}_{m}$ denotes the ML detected symbol. By (7), the two-main steps of the ML for CDIDM are (1) compute $\bar{D}_{M L}=\left\|\mathbf{y}_{B}-\boldsymbol{c}_{i} b_{m}\right\|^{2}$ for all combinations of the modulated symbol $b_{m}$ and spreading codes; (2) select the modulation symbol and spread code index corresponding to the smallest $\bar{D}_{M L}$ as the final detected result.

2) Eve's Detection: The signal received at Eve can be written as

$$
\begin{aligned}
\mathbf{z} & =\mathbf{H}^{H}\left(\Theta_{E}\right) \mathbf{x}+\mathbf{n} \\
& =\mathbf{H}^{H}\left(\Theta_{E}\right) \mathbf{P} \boldsymbol{c}_{i} b_{m}+\mathbf{n},
\end{aligned}
$$




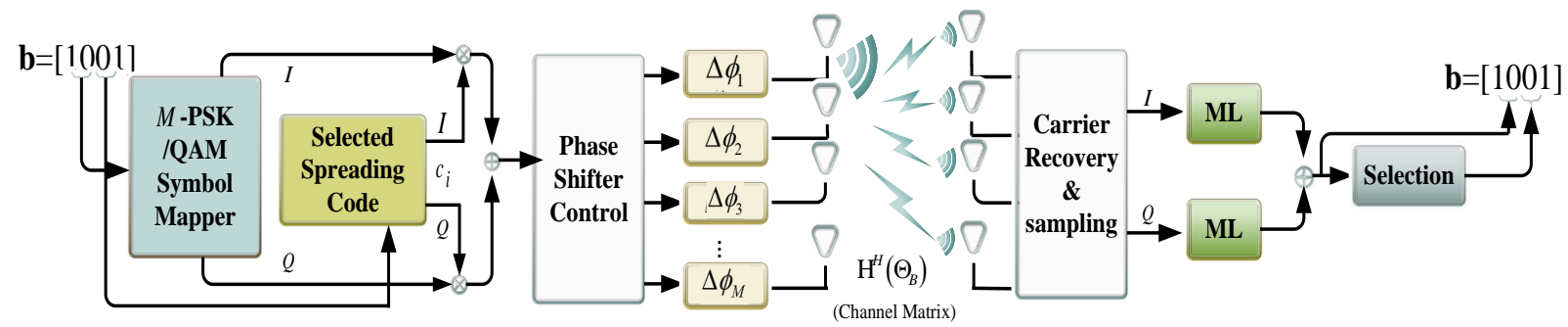

Fig. 2. Transceiver architecture of CDIDM

where $\Theta_{E}$ is the direction of Eve, and $\mathbf{H}^{H}\left(\Theta_{E}\right)$ is the channel matric from Alice to Eve. Assuming Eve has unlimited computational capabilities, Eve can use the ML detector to detect $M$-PSK/QAM symbols $\hat{b}_{m}$ as

$$
\left\langle\hat{b}_{m}\right\rangle=\arg \min _{b_{m} \in \mathcal{B}}\left\|\mathbf{z}-\boldsymbol{c}_{i} b_{m}\right\|^{2} .
$$

On one hand, due to the introduction of the CIM, if Eve does not know the spread code set used by Alice and Bob, then it is almost impossible for Eve to recover the original information. On the other hand, even if Eve knows the spread code set that Alice and Bob used, (9) shows that the presence of the scrambling factor $\mathbf{H}^{H}\left(\Theta_{E}\right) \mathbf{P}$ reduces the detection performance of $b_{m}$ since it is difficult for Eve to obtain the channel state information (CSI) between Alice and Bob. Thus, CIDDM can achieve high levels of security.

In this letter, the detection complexity of the CDIDM scheme is evaluated by the number of spreading code required to transmit a symbol. In general, the CDIDM scheme requires two spreading operations to transmit one symbol in the IQ-channel, which is the same as that required in the case of CIM. Moreover, based on the complexity analysis framework for CIM given in [9], in the proposed CDIDM, $N$ spreading codes represent $N$ complex operations for each detection symbol in $(7)$ and hence the detection complexity of CDIDM system is approximated to $\mathcal{O}_{C I M}=2 \times 2^{K M} \times N$, where $K$ is the length of spreading code.

\section{BER PERFormanCe ANALysis}

In this section, the joint ABEP boundaries of Bob and Eve are obtained under the assumption of ML detection.

\section{A. BER Analysis}

According to the CDIDM system, we define $P_{T}$ as the total BER probability of the system, $P_{e b}$ the BER probability of the modulated bits, and $P_{e m}$ the BER probability of the spread code index mapped bits. Thus, the overall BER probability of the CDIDM system is given by

$P_{T}=\frac{\log _{2}(M)}{\log _{2}(M)+\left\lfloor\log _{2}(N)\right\rfloor} P_{e b}+\frac{\left\lfloor\log _{2}(N)\right\rfloor}{\log _{2}(M)+\left\lfloor\log _{2}(N)\right\rfloor} P_{e m}$,

where $P_{\text {em }}$ depends on the number of mapped bits $\left\lfloor\log _{2}(N)\right\rfloor$. Each possible combination may have a different number of error bits compared to the correct combination of bits. The probability of detecting the remaining $N-1$ error combinations is the same for all codes which is equal to $1 /(N-1)$. Thus, the BER probability of the mapped bits can be expressed as

$$
P_{e m}=\frac{P_{e d}}{\left\lfloor\log _{2}(N)\right\rfloor(N-1)} \sum_{c=1}^{\left\lfloor\log _{2}(N)\right\rfloor}\left(\begin{array}{c}
\left\lfloor\log _{2}(N)\right\rfloor \\
c
\end{array}\right),
$$

where $\left(\begin{array}{c}\left\lfloor\log _{2}(N)\right\rfloor \\ c\end{array}\right)$ denotes the number of $c$-bit combinations that are different from the correct bit combination, and the BER associated with error code detection is $P_{e d}$. It is easy to see that by calculating the BER probability of the mapped bit $P_{e b}$ and the BER probability of the modulated bit $P_{e m}$, one can obtain the total probability of the BER $P_{T}$. Here, $P_{e m}$ has been calculated in (11), and the BER probability of the modulated bit $P_{e b}$ will be discussed below.

Note that the correct original bits rely on code detection and $M$-ary demodulation. Thus, errors may occur in two distinct cases. In the case of (1), it is correct during the spread code detection, but an error occurred during the modulation symbol detection. For (2), there is an error in the code detection, and the modulated symbol is detected based on the error detection of spread spectrum code. Consequently, the BER probability of the modulated bit can be stated as

$$
P_{e b}=P_{s s}\left(1-P_{e m}\right)+\frac{1}{2} P_{e m} .
$$

where $P_{s s}$ stands for the BER of the conventional direct-sequence spread spectrum $M$-ary system in [23], which depends on the number of modulation bits, and noted that $P_{e m}$ has been accounted in (11). Factor $(1 / 2)$ indicates that in the case of an error in the mapped bit detection, the detected bits based on the incorrect demodulation symbol still have a half-chance of matching the original modulation bits. It is clear that both (10) and (12) are computable as soon as the error probability of spreading code detection of $P_{e d}$ is obtained.

\section{B. Error Detection Probability $P_{e d}$}

In this subsection, we analyze the derivation of the error code detection probability $P_{e d}$ which is needed to calculate the BER. We use the detection method of the CIM receiver to calculate the BER of the detection part of spread code in the CDIDM system. For equally likely transmission of spreading codes, the error probability conditional on the spreading code $\boldsymbol{c}_{\hat{i}}$ and the channel coefficient $\mathbf{h}(\theta)$ is

$$
P_{e d}=\operatorname{Pr}\left(\left|I_{\hat{i}}\right|<\min _{\substack{i \in\{1,2, ., N-1\} \\ i \neq \hat{i}}}\left\{\left|I_{i}\right|\right\} \mid\left\{\boldsymbol{c}_{i}, \mathbf{h}(\theta)\right\}\right),
$$




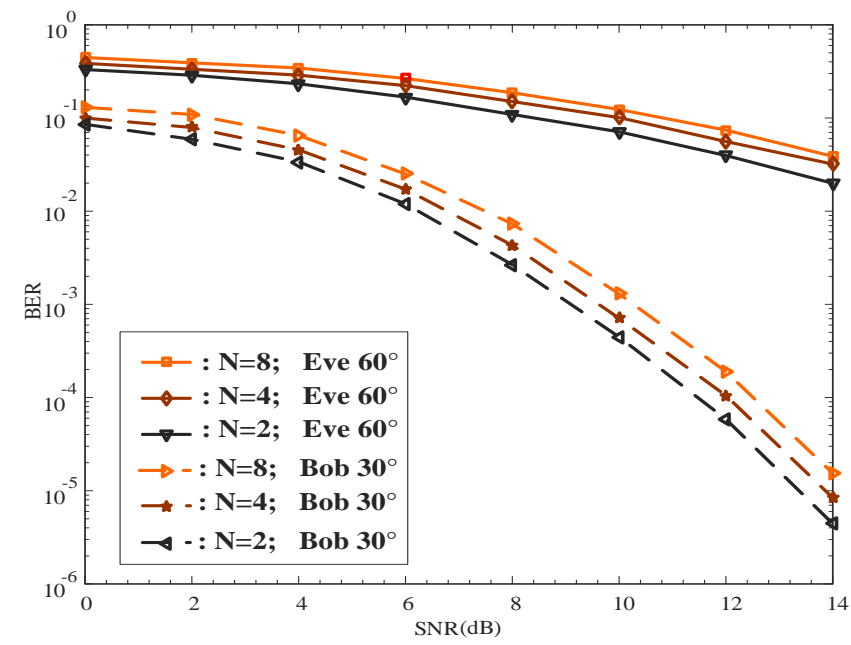

Fig. 3. BER performance of CDIDM with the different numbers of spreading codes when Bob and Eve are $60^{\circ}$ and $30^{\circ}$, respectively.

where $I_{\hat{i}}= \pm E_{s}|\mathbf{h}(\theta)|^{2}+v_{I, \hat{i}}$ and $I_{i}=v_{I, i} . \quad E_{s}=\sum_{l=1}^{k} c_{\hat{i}, l}^{2}$ is the spreading code energy. Since different random variables $I_{i}$ are independent and obey the folded normal distribution, the theory of ordered statistics can be applied to calculate (13). Since

$$
\operatorname{Pr}\{Y<X\}=\int_{0}^{\infty} \prod_{q=1}^{N-1} \operatorname{Pr}\left\{y<X_{q}\right\} f_{Y}(y) d y
$$

(13) is calculated as

$$
\begin{aligned}
& P_{e d}=\frac{1}{\sqrt{\pi \sigma_{Y}^{2}}} \int_{0}^{\infty}\left[1-\operatorname{erf}\left(\frac{y}{\sqrt{2} \sigma_{Y}}\right)\right]^{K} \\
& \left.\times e^{-\frac{(y-E\{Y\})^{2}}{2 \sigma_{Y}^{2}}}+e^{-\frac{(y+E\{Y\})^{2}}{2 \sigma_{Y}^{2}}}\right) d y,
\end{aligned}
$$

and can be further written as

$$
\begin{aligned}
P_{e d}= & \left.\frac{1}{|\mathbf{h}(\theta)|^{2} \sqrt{\pi \rho}} \int_{0}^{\infty}\left[1-\operatorname{erf} \frac{y}{|\mathbf{h}(\theta)|^{2} \sqrt{2 \rho}}\right)\right]^{K} \\
& \left.\times \quad e^{-\frac{\left(y-\beta|\mathbf{h}(\theta)|^{2}\right)}{2 \rho|\mathbf{h}(\theta)|^{4}}}+e^{-\frac{\left(y+\beta|\mathbf{h}(\theta)|^{2}\right)}{2 \rho|\mathbf{h}(\theta)|^{4}}}\right) d y,
\end{aligned}
$$

where $\beta$ and $\rho$ are expressed in (17) and (18) as follows:

$$
\beta=\sqrt{\frac{E_{s} N_{0}}{2 \pi} e^{\left(-\frac{E_{s}}{2 N_{0}}\right)}-\frac{E_{s}}{2} \operatorname{erf}\left(-\frac{E_{s}}{2 N_{0}}\right)},
$$

$\left.\rho=\frac{E_{s}}{4}\left(E_{s}+N_{0}\right)-\sqrt{\frac{E_{s} N_{0}}{2 \pi}} e^{-\frac{E_{s}}{2 N_{0}}}-\frac{E_{s}}{2} \operatorname{erf}\left(-\frac{E_{s}}{2 N_{0}}\right)\right)^{2}$.

In addition, $K=N-1, \mathbf{h}(\theta)$ indicates channel coefficient and erf $=\frac{2}{\sqrt{\pi}} \int_{0}^{x} e^{-\eta^{2}} d \eta$ is the error function. Finally, the average error detection probability $P_{e d}$ is equal to

$$
P_{e d}=\int_{0}^{\infty} \int_{0}^{\infty} P_{e d} f_{\mathbf{h}(\theta)}(\alpha) d y d \alpha
$$

where $f_{h}(\alpha)$ is the probability distribution function of the channel gain. Since the closed-form expression is not accessible, we can easily simplify the above integral with numerical integration by using Mathematica or Matlab, and calculate the probability of error detection with numerical integration as the solution. Finally,

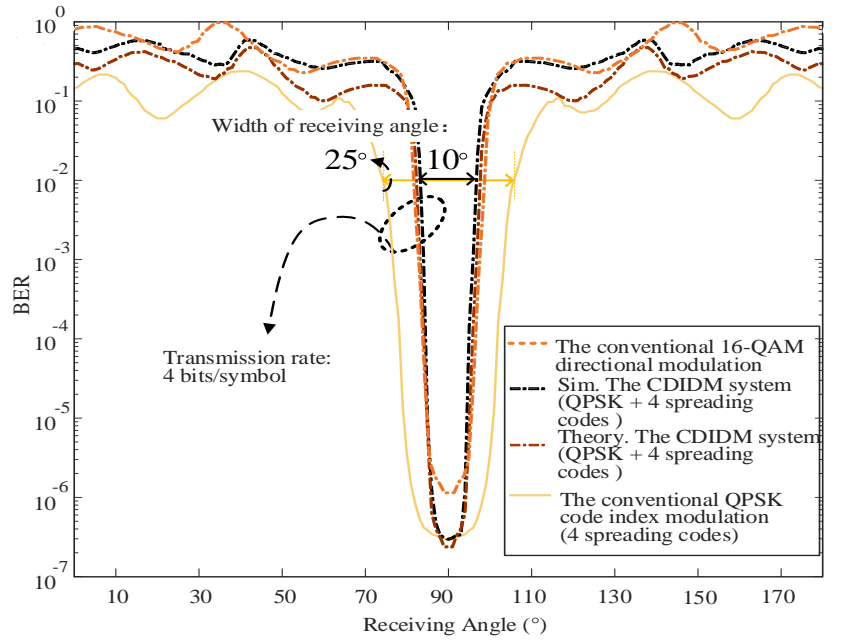

Fig. 4. BER when desired $\theta=90^{\circ}$ is at broadside for CIM, DM, and CDIDM simulated BER

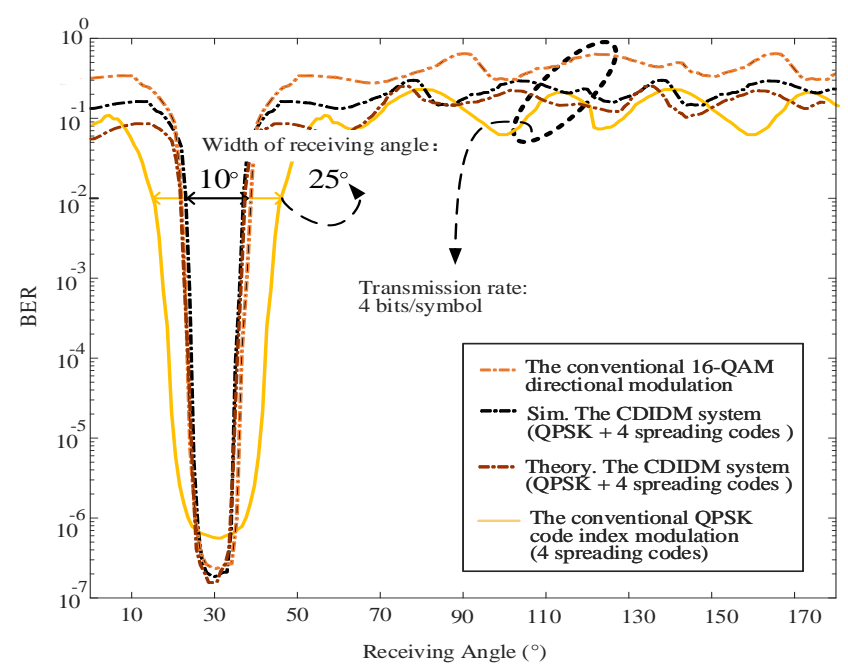

Fig. 5. BER when desired $\theta=30^{\circ}$ is at broadside for CIM, DM, and CDIDM simulated BER

by substituting (19) into (11) to get $P_{\text {em }}$, and then substituting (11) and (12) into (10), the total BER of the CDIDM system in the fading channel can be obtained.

In combining directional modulation, Bob and Eve need to be discussed one by one due to the effect of angle on the channel matrix. For the Bob case, the channel coefficient can be equivalent to 1 (i.e., $\mathbf{h}(\theta)=1$ ). Thus, the closed expression for the error probability $P_{e d}$ can be obtained directly from (13), and the total BER under AWGN channel can be obtained by substituting $\mathbf{h}(\theta)=1$ and (16) in (12) into (9). But for Eve, the difference is that the equivalent channel coefficient is equal to $\mathbf{H}^{H}\left(\Theta_{E}\right) \mathbf{P}$. We then need to bring in the specific wrong azimuth $\Theta_{E}$ to calculate $|\mathbf{h}(\theta)|^{2}$ at this point.

\section{Simulation Results and Analysis}

In this section, numerical simulation results are presented to demonstrate the effect of the spreading codes and angle on the receiver side in the CDIDM system. For this purpose, we consider the number of antennas in Alice is $N_{t}=4$, set SNR as $12 \mathrm{~dB}$, and the performance of the CDIDM system is under LoS channel conditions. 
Fig. 3 shows the achievable BER performance versus SNR for different numbers of spreading codes. The desired direction $\theta$ means that the receiving angle corresponds to the transmitting angle. As seen in Fig. 3, the BER for Bob and Eve reduces with decreasing $N$ for a given SNR. The reason for this is that as the number of spreading codes increasing, there is more potential for errors in the detection of spreading codes at the end of the receiver. However, the transmission rate can be improved by increasing the number of spreading codes within the acceptable BER.

On the one hand, the angle having BER below $10^{-2}$ is defined as the range of correct receiving angle (RCA), which is used to evaluate the concealment performance of the system. The narrower the angle width of the receiving, the narrower the range of correct receiving angle, and the better the concealment of the system. On the other hand, the BER with high safety performance should be as high as possible in the undesired direction, and the more stable the better. Therefore, we define the evaluation criteria for the stability of the BER in the undesired direction, that is, the variance is used to represent the stability of the error rate in the undesired direction.

$$
\mathbb{D}\left(P_{\text {ber }}(\theta)\right)=\mathbb{E}\left(\left\{\left|P_{\text {ber }}(\theta)-\mathbb{E}\left\{P_{\text {ber }}(\theta)\right\}\right|^{2}\right\}\right), \theta \in U A,
$$

where $P_{\text {ber }}(\theta)$ is the BER of receiving angel $\theta$ and $U A$ is the set of unexpected angels.

Fig. 4 and Fig. 5 respectively show the simulated BER performance of the CIM, DM and CDIDM schemes when the desired angles are $\theta=90^{\circ}$ and $\theta=30^{\circ}$. Maintaining the same transmission rate (i.e. $\left.\log _{2}(M)+\left\lfloor\log _{2}(N)\right\rfloor=4\right)$, the RCA of the CDIDM can achieve $10^{\circ}$, which is narrower than the RCA of the CIM. Furthermore, by calculating the $\mathbb{D}\left(P_{b e r}(\theta)\right)$ of the three systems, $\mathbb{D}\left(P_{\text {ber }}(\theta)\right)$ of the CDIDM is 0.0086 while $\mathbb{D}\left(P_{b e r}(\theta)\right)$ of the $\mathrm{DM}$ is 0.0255 and $\mathbb{D}\left(P_{b e r}(\theta)\right)$ of the CIM is 0.0104 . The CDIDM maintain a higher and more stable BER at the unexpected angle. Finally, the ABEP of theoretical analysis is very close to the simulation results, which verifies that the $\mathrm{ABEP}$ is close to the actual simulation results within the allowable difference range. Therefore, ABEP has reference value for future research.

\section{CONClusion}

In this paper, we have proposed a hybrid multi-domain index modulation system with good BER performance and a high data rate for future covert communications. Our extensive simulation results have shown that the proposed CDIDM scheme is able to not only transmit information covertly by using spreading codes index, but also maintain a low BER over an angular range of about $10^{\circ}$.

\section{REFERENCES}

[1] M. Au, G. Kaddoum, M. S. Alam, E. Basar, and F. Gagnon, "Joint code-frequency index modulation for IoT and multi-user communications," IEEE Journal of Selected Topics in Signal Processing, vol. 13, no. 6, pp. 1223-1236, Oct. 2019.

[2] E. Basar, $\ddot{U}$. Aygöl $\ddot{u}$, E. Panaylrcl, and H. V. Poor, "Orthogonal frequency division multiplexing with index modulation," IEEE Trans. Signal Process., vol. 61, no. 22, pp. 5536-5549, Nov. 2013.
3] S. Sugiura, T. Ishihara, and M. Nakao, "State-of-the-Art design of index modulation in the space, time, and frequency domains: benefits and fundamental limitations," IEEE Access, vol. 5, pp. 21774-21790, 2017.

[4] Q. D. You and Y. P. Xiao, "Spatial and directional modulation with scrambling," Phys. Commun., vol. 35, Aug. 2019.

5] L. Wang, S. Bashar, Y. Wei, and R. Li, "Secrecy enhancement analysis against unknown eavesdropping in spatial modulation," IEEE Commun.Lett., vol. 19, no. 8, pp. 1351-1354, Aug. 2015.

[6] Z. Huang, Z. Gao, and L. Sun, "Anti-eavesdropping scheme based on quadrature spatial modulation," IEEE Commun. Lett., vol. 21, no. 3, pp. 532-535, Mar. 2017.

[7] C. Wu, Y. Xiao and P. Yang, "Covert information embedded spatial modulation," IEEE Commun. Lett., vol. 24, no. 11, pp. 2426-2430, Nov. 2020

[8] G. Kaddoum, M. F. A. Ahmed, and Y. Nijsure, "Code index modulation: A high data rate and energy efficient communication system," IEEE Commun. Lett., vol. 19, no. 2, pp. 175-178, Feb. 2015.

[9] F. Cogen, E. Aydin, N. Kabaoglu, E. Basar and H. Ilhan, "Generalized code index modulation and spatial modulation for high rate andenergy-efficient MIMO systems on rayleigh block-fading channel," IEEE Systems Journal, vol. 15, no. 1, pp. 538-545, Mar. 2021.

[10] E. Aydin, F. Cogen and E. Basar, "Code-Index modulation aided quadrature spatial modulation for high-rate MIMO systems," IEEE Transactions on Vehicular Technology, vol. 68, no. 10 , pp. 10257-10261, Oct. 2019.

[11] F. Cogen, E. Aydin, N. Kabaoglu, E. Basar and H. Ilhan, "Code index modulation and spatial modulation: A new high rate and energy efficient scheme for MIMO systems," in Proc. 41 st Int. Conf. Telecommun. Signal Process (TSP), Athens, Greece, 2018, pp. 1-4.

[12] E. Aydin and F. Cogen, "Two-Way code index modulation," in proc. 27th Signal Processing and Communications Applications Conference (SIU), Sivas, Turkey, 2019, pp. 1-4.

[13] Y. Ding and V. Fusco, "A review of directional modulation technology," Int. J. Microw. Wireless Technol., vol. 8, no. 7, pp. 1-13, Jul. 2015.

[14] M. Hafez and H. Arslan, "On directional modulation: an analysis of transmission scheme with multiple directions," in Proc. IEEE Int. Conf. Commun. Workshop (ICCW), London, U.K., Jun. 2015, pp. 459-463.

[15] P. Antonik, M. C. Wicks, H. D. Griffiths and C. J. Baker, "Frequency diverse array radars," in Proc. IEEE Conf. Radar, Verona, NY, USA, Apr. 2006, pp. 215-217.

[16] W. Khan, I. M. Qureshi and S. Saeed, "Frequency diverse array radar with logarithmically increasing frequency offset," IEEE Antennas Wireless Propag. Lett., vol. 14, pp. 499-502, Nov. 2015.

[17] H. Shao, J. Dai, J. Xiong, H. Chen and W.-Q. Wang, "Dot-shaped range-angle beampattern synthesis for frequency diverse array," IEEE Antennas Wireless Propag. Lett., vol. 15, pp. 1703-1706, Feb. 2016.

[18] Y. Liu, H. Ruan, L. Wang and A. Nehorai, "The random frequency diverse array: a new antenna structure for uncoupled direction-range indication in active sensing," IEEE J. Sel. Topics Signal Process., vol. 11, no. 2, pp. 295-308, Mar. 2017.

[19] S. Y. Nusenu, W.-Q. Wang and S. Ji, "Secure directional modulation using frequency diverse array antenna," in Proc. IEEE Radar Conf., Seattle, WA, USA, May 2017, pp. 378-382.

[20] J. Xiong, S. Y. Nusenu and W.-Q. Wang, "Directional modulation using frequency diverse array for secure communications", Wireless Pers. Commun., vol. 95, no. 3, pp. 2679-2689, Aug. 2017.

[21] W.-Q. Wang, "DM using FDA antenna for secure transmission," IET Microw. Antennas Propag., vol. 11, no. 3, pp. 336-345, 2017

[22] J. Hu, S. Yan, F. Shu, J. Wang, J. Li and Y. Zhang, "Artificial-noise-aided secure transmission with directional modulation based on random frequency diverse arrays," IEEE Access, vol. 5, pp. 1658-1667, 2017.

[23] Q. D. You and Y. P. Xiao, "Spatial and directional modulation with scrambling," Phys. Commun., vol. 35, Aug. 2019.

[24] R. Y. Mesleh, H. Haas, S. Sinanovic, C. W. Ahn, and S. Yun, "Spatial modulation," IEEE Trans. Veh. Technol., vol. 57, no. 4, pp. 2228-2241, Jul. 2008. 